\title{
Visualizing Changes in Circuit Activity Resulting from Denervation and Reinnervation Using Immediate Early Gene Expression
}

\author{
Meredith D. Temple, ${ }^{1}$ Paul F. Worley, ${ }^{2}$ and Oswald Steward ${ }^{1}$ \\ ${ }^{1}$ Reeve-Irvine Research Center, Departments of Anatomy and Neurobiology, and Neurobiology and Behavior, University of California at Irvine, Irvine, \\ California 92697, and 2Departments of Neuroscience and Neurology, Johns Hopkins University, Baltimore, Maryland 21205
}

We describe a novel strategy to evaluate circuit function after brain injury that takes advantage of experience-dependent immediate early gene (IEG) expression. When normal rats undergo training or are exposed to a novel environment, there is a strong induction of IEG expression in forebrain regions, including the hippocampus. This gene induction identifies the neurons that are engaged during the experience. Here, we demonstrate that experience-dependent IEG induction is diminished after brain injury in young adult rats (120 -200 $\mathrm{gm})$, specifically after unilateral lesions of the entorhinal cortex (EC), and then recovers with a time course consistent with reinnervation. In situ hybridization techniques were used to assess the expression of the activity-regulated cytoskeleton-associated protein Arc at various times after the lesion $(4,8,12,16$, or $30 \mathrm{~d})$. One group of rats was allowed to explore a complex novel environment for $1 \mathrm{hr}$; control operated animals remained in their home cage. In unoperated animals, exposure to the novel environment induced Arc mRNA levels in most pyramidal neurons in CA1, in many pyramidal neurons in CA3, and in a small number of dentate granule cells. This characteristic pattern of induction was absent at early time points after unilateral EC lesions ( 4 and $8 \mathrm{~d}$ ) but recovered progressively at later time points. The recovery of Arc expression occurred with approximately the same time course as the reinnervation of the dentate gyrus as a result of postlesion sprouting. These results document a novel approach for quantitatively assessing activity-regulated gene expression in polysynaptic circuits after trauma.

Key words: immediate early gene; reinnervation; denervation; recovery of function; sprouting; entorhinal cortex; hippocampus; fimbriafornix; diaschisis; rat; Arc

\section{Introduction}

CNS injury disrupts synaptic circuitry, which is likely to cause widespread disruptions of information processing. Injury also often triggers synaptic reorganization, and this reorganization may contribute to recovery of function (Steward, 1982). To relate changes in synaptic circuitry to behavioral deficits and subsequent recovery, it is important to determine how injury and subsequent synaptic reorganization affects neuronal activity in widespread brain circuits.

A case in point is the example of synaptic reorganization that occurs in the hippocampal dentate gyrus after damage to the entorhinal cortex (EC) (Steward, 1989). Unilateral lesions of the EC cause the degeneration of synapses that terminate on the distal dendrites of granule cells in the ipsilateral dentate gyrus, eliminating the main cortical input to the hippocampus of one hemisphere (Matthews et al., 1976a; Steward and Vinsant, 1983; Steward, 1989). Over time, the granule cells are reinnervated as a consequence of the sprouting of surviving afferent systems (Mat-

Received Dec. 3, 2002; revised Jan. 13, 2003; accepted Jan. 14, 2003.

This work was supported by National Institutes of Health Grant NS32280 (0.S.). M.D.T. was the recipient of National Research Service Award Training Grant HD007323.

Correspondence should be addressed to Dr. Oswald Steward, Reeve-Irvine Research Center, 1105 Gillespie Neuroscience Research Facility, 837 Health Sciences Drive, University of California at Irvine, Irvine, CA 92697. E-mail: osteward@uci.edu.

M. D. Temple's present address: National Institute of Biomedical Imaging and Bioengineering, Democracy II, Suite 200, Mail Stop Code 5477, 6707 Democracy Boulevard, Bethesda, MD 20892.

Copyright $\odot 2003$ Society for Neuroscience $\quad 0270-6474 / 03 / 232779-10 \$ 15.00 / 0$ thews et al., 1976b; Steward and Vinsant, 1983). Neurophysiological studies of the synaptic properties of the new connections that form in response to lesions have revealed physiological properties that are not fundamentally different from the normal synapses (Steward, 1982). Also, studies of the firing characteristics of dentate granule cells reveal that denervation leads to decreases in the "spontaneous activity" (average rate of firing in anesthetized animals) of dentate granule cells and that spontaneous activity recovers with reinnervation (Reeves and Steward, 1988). Behavioral studies have revealed deficits after EC lesions that recover with a time course that is also similar to the time course of reinnervation (Loesche and Steward, 1977; Phillips et al., 1994). The temporal relationship between synaptic reorganization and behavioral recovery suggests a relationship, but it is not known how the reinnervating fibers actually affect the functional activity of the hippocampus and its related circuitry as these neurons are engaged in their normal functional activities.

To address this question, it is important to have a means to assess the activity of ensembles of neurons during particular behavioral experiences. The present study described a novel way to do this that takes advantage of the fact that immediate early gene (IEG) expression, more particularly the expression of activityregulated cytoskeleton-associated gene $A r c$, is strongly upregulated in a cell type-specific manner by behavioral experiences (Guzowski et al., 1999; Pinaud et al., 2001; Kelly and Deadwyler, 2002). This IEG induction is thought to reflect the fact that particular ensembles of neurons are engaged during the behavioral 
experience. We reasoned that assessment of experience-induced IEG expression might be useful to define the ensembles of neurons that were activated by experience in brains in which connections had been modified as a consequence of lesions. We show here that unilateral EC lesions profoundly disrupt experiencedependent induction of IEG expression in areas that were directly denervated (the ipsilateral dentate gyrus) and in related circuits. Activity-induced IEG expression then recovered at approximately the same time that reinnervation occurs. Comparison of activity-induced Arc expression after destruction of different hippocampal afferent pathways indicated that information important for the induction of Arc during a behavioral experience comes from multiple inputs.

\section{Materials and Methods \\ Animals}

Experimental animals were male Sprague Dawley rats (Hilltop, Scottsdale, PA) weighing 120-200 gm. Animals were housed in individual cages, with food and water available ad libitum. The animal colony was maintained at a temperature of $20-22^{\circ} \mathrm{C}$ with a $12 \mathrm{hr}$ light/dark cycle (lights on at 6:00 A.M. and lights off at 6:0 P.M.).

\section{Comparing IEG expression after different behavioral experiences}

Previous studies demonstrated that different types of brief behavioral experience induce IEG expression and especially the expression of Arc in forebrain structures, including the hippocampus (Guzowski et al., 1999; Pinaud et al., 2001; Kelly and Deadwyler, 2002). To assess alterations in experience-induced gene expression with denervation and reinnervation, it is desirable to use an experiential paradigm that induces robust IEG expression in the structures that are actually denervated, as well as in related circuitry. Accordingly, in an initial study, we compared the pattern of IEG induction in the dentate gyrus and downstream structures, including the $\mathrm{CA} 3$ and $\mathrm{CA} 1$ regions of the hippocampus, after two types of brief experience, exposure to a novel complex environment (a toyfilled arena) for $1 \mathrm{hr}$ versus explicit training in the Morris water maze (MWM). The former offers the advantage that the manipulation is simple, but the experience is less structured because it depends on the animal's exploratory activity. Training in the MWM has the advantage that it is an explicit learning paradigm, the results of which can be assessed by measuring recall. The disadvantage is that the task is less convenient to use as a routine assessment tool. Also, the nature of the experience changes with repetition as the animals learn the task (which would be expected to produce different patterns of IEG induction).

Forty-six male Sprague Dawley rats were used (120-200 gm from Hilltop). Animals were divided into four groups as follows: (1) isolated home cage control, in which the animals were simply left in the home cage; (2) exposure to a novel enriched environment, in which animals were placed in the novel enriched environment (two to three at a time) consisting of a $3 \times 3$ foot box with toys of different textures and shapes and were allowed to explore freely for $1 \mathrm{hr}$; (3) one training session (four sequential trials) in the Morris water maze; and (4) four training sessions (four trials each) in the Morris water maze over a $4 \mathrm{~d}$ period.

The MWM was a galvanized steel stock tank (3 foot diameter) painted white and filled with water to a depth of $27.5 \mathrm{~cm}$. The water was made opaque with nontoxic paint. A Plexiglas platform $(25.5 \mathrm{~cm}$ high, which would be $2 \mathrm{~cm}$ below the water surface) was placed in the southeast quadrant of the tank, and animals were trained in a room with constant extramaze cues. Training consisted of four trials per session. The trials were initiated by placing the rat in one of the four quadrants of the maze (one trial starting in each quadrant, with order chosen randomly for each four trial training session). On a given trial, animals were allowed $60 \mathrm{sec}$ to locate the hidden platform and then were allowed to remain on it for $15 \mathrm{sec}$. If they did not locate the platform, they were placed on it for $15 \mathrm{sec}$ and then removed, dried, and returned to their home cage for a $5 \mathrm{~min}$ intertrial rest interval.

After the $1 \mathrm{hr}$ exposure to the novel complex environment, or $1 \mathrm{hr}$ after the training session in the MWM, animals received an anesthetic overdose (sodium pentobarbital) and were perfused transcardially with $4 \%$ paraformaldehyde in $0.1 \mathrm{~m}$ phosphate buffer (PB), $\mathrm{pH}$ 7.4. Brains were removed, kept in fixative overnight at $4^{\circ} \mathrm{C}$, and then were transferred to $30 \%$ sucrose overnight for cryoprotection. The brains of home cage control and experimental animals were blocked in the coronal plane, embedded together in a single mold using OCT, and frozen using liquid nitrogen. Coronal sections were cut on a cryostat ( $20 \mathrm{~mm}$ thickness), mounted on poly-L-lysine ( $1 \mathrm{mg} / \mathrm{ml}$; Sigma, St. Louis, MO) -treated slides and stored at $-80^{\circ} \mathrm{C}$ until in situ hybridization was performed.

\section{Assessment of IEG expression in brain-injured animals}

Surgery. Animals to receive lesions $(n=57)$ were anesthetized with sodium pentobarbital $(50 \mathrm{mg} / \mathrm{kg}$ ). Electrolytic lesions of the right EC were produced, as described previously (Loesche and Steward, 1977). Unilateral lesions of the EC denervate granule cells in the ipsilateral dentate gyrus and trigger a reinnervation process that involves a number of surviving afferent systems, including one from the opposite EC. Controls included unoperated animals $(n=24)$ and sham-operated controls $(n=$ 6) that were anesthetized and prepared for surgery without electrode penetration.

Because the reinnervating fibers from the opposite hemisphere are likely to carry the same type of information as the normal fibers that they replace, it was of interest to assess experience-induced IEG expression in animals in which the reinnervation by the contralateral entorhinal cortex was prevented. For this purpose, another group of animals received bilateral entorhinal cortex lesions $(n=12)$.

Another group of animals $(n=14)$ received unilateral fimbria-fornix transactions to define the consequences of eliminating a different type of input to the dentate gyrus and hippocampus. Animals were anesthetized as above, and the fimbria-fornix was transected unilaterally using an ophthalmic surgical blade. The blade was lowered into the brain $1 \mathrm{~mm}$ posterior to bregma to a depth of $4.5 \mathrm{~mm}$ (blade pointing laterally) and was then moved from midline $(0 \mathrm{~mm})$ to $4 \mathrm{~mm}$ laterally along a track that was at a $30^{\circ}$ angle with respect to the midline.

Exploration in the novel environment. Animals with unilateral EC lesions were assigned to one of five survival groups: 4, 8, 12, 16, or $30 \mathrm{~d}$ after lesion (Table 1). Animals with bilateral EC lesions or unilateral fimbriafornix transections (and their respective control groups) were assigned to one of two survival groups ( 4 or $30 \mathrm{~d}$ after lesion). On the day the animals were to be killed, one group of animals was allowed to explore the novel complex environment for $1 \mathrm{hr}$ (the exploration group) and then received an overdose of sodium pentobarbital. The control animals remained in their home cage (isolated group) and were anesthetized without any intervening behavioral experience.

Animals were perfused transcardially with $4 \%$ paraformaldehyde in $0.1 \mathrm{M} \mathrm{PB}, \mathrm{pH} 7.4$, and brains of both isolated and "enriched" animals (with and without lesions) were blocked in the coronal plane portions at approximately the posterior boundary of the hippocampus, embedded together in a single mold using OCT, and rapidly frozen using liquid nitrogen. Coronal sections were cut on a cryostat (20 mm thickness), mounted on poly-L-lysine ( $1 \mathrm{mg} / \mathrm{ml}$; Sigma $)$-treated slides and stored at $-80^{\circ} \mathrm{C}$ until in situ hybridization was performed.

Lesion verification. The posterior portion of the brains from the animals with unilateral or bilateral EC lesions was cryoprotected, frozen in OCT, and cut on a cryostat ( $20 \mu \mathrm{m}$ thickness) in the horizontal plane. One section every $400 \mu \mathrm{m}$ was collected and stained with cresyl violet to verify the location and extent of the lesion. In addition, coronal sections from the anterior portions of the brains were stained for acetylcholinesterase (AChE). Increases in AChE staining mark the areas of the molec-

Table 1. Numbers of animals with complete versus selective medial lesions at the different postlesion intervals

\begin{tabular}{|c|c|c|c|c|c|}
\hline & $4 d$ & $8 d$ & $12 d$ & $16 \mathrm{~d}$ & $30 d$ \\
\hline \multicolumn{6}{|l|}{ Isolated } \\
\hline Complete & $n=5$ & $n=2$ & $n=1$ & $n=0$ & $n=2$ \\
\hline Selective medial & $n=1$ & $n=5$ & $n=4$ & $n=6$ & $n=3$ \\
\hline \multicolumn{6}{|l|}{ Enriched } \\
\hline Complete & $n=5$ & $n=0$ & $n=2$ & $n=2$ & $n=5$ \\
\hline Selective medial & $n=1$ & $n=6$ & $n=3$ & $n=6$ & $n=2$ \\
\hline
\end{tabular}



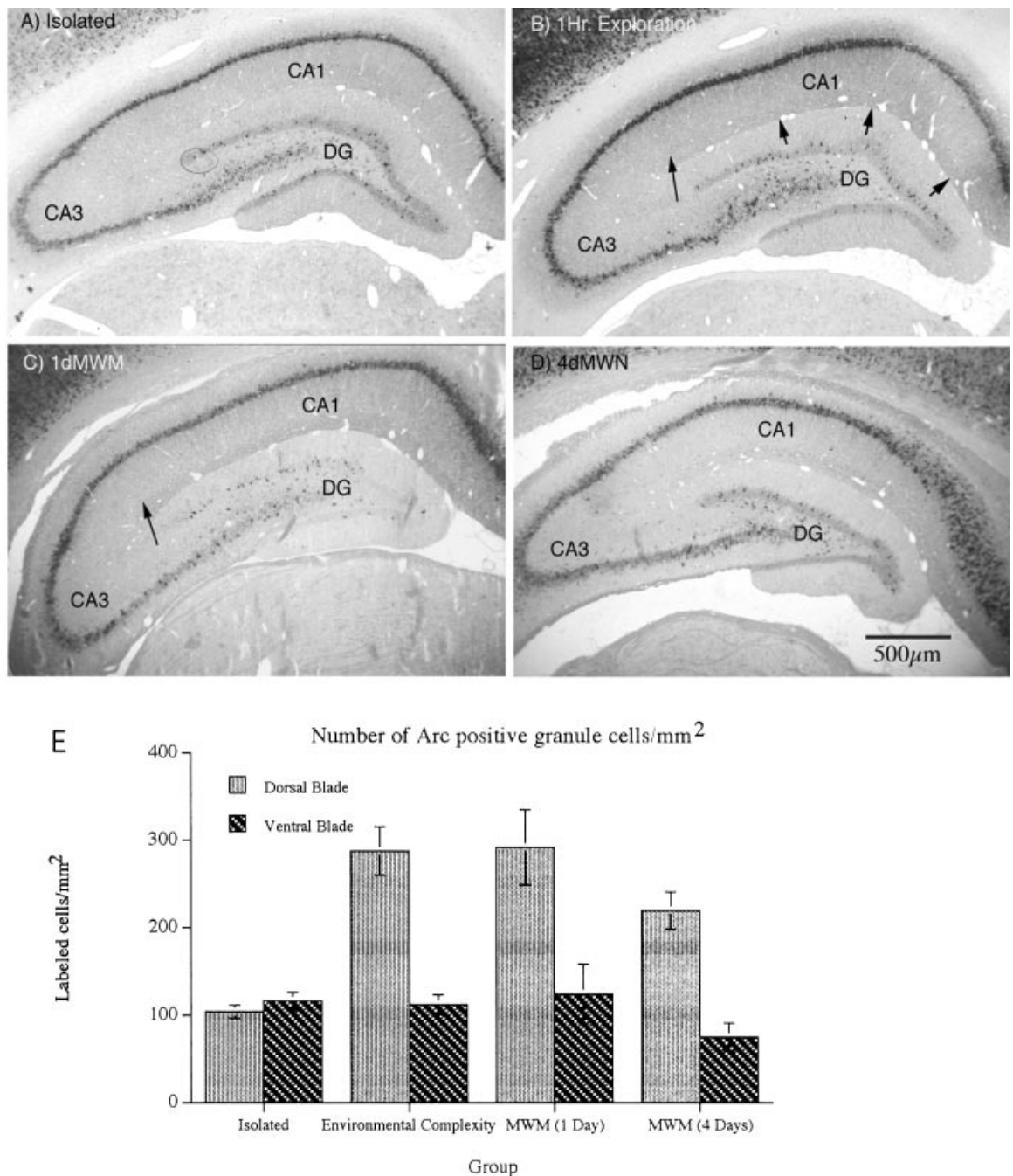

Figure 1. Induction of Arc expression after different behavioral experiences. The photomicrographs illustrate Arc mRNA distribution as revealed by nonisotopic in situ hybridization. $A$, Control animals left undisturbed in their home cage (isolated controls). $B$, After $1 \mathrm{hr}$ of exploration of a novel complex environment. C, One hour after four trials (1 session) of training in a Morris water maze. $D$, One hour after the last of $4 \mathrm{~d}$ of training ( 4 trials per day) in a Morris water maze. Note the experience-induced increases in labeling in both the cell layers and dendritic layers of the CA1 region of the hippocampus and the increase in the number of dentate granule cells exhibiting high levels of labeling. $C A 1$ and $C A 3$ indicate the respective divisions of the hippocampus; $D G$ refers to granule cell layer, in which heavily labeled granule cells can be seen. Short arrows in $B$ indicate labeled dendrites. Long arrows indicate the boundary of dendritic labeling at the $C A 1 / C A 2$ junction. $E$, The graph plots the average numbers of Arc-positive dentate granule cells per square millimeter in the different groups. Note the substantial increases in the number of labeled granule cells in the dorsal blade in each of the experimental groups.

ular layer in which sprouting occurs, providing another measure of the completeness of the EC lesions.

In situ hybridization. The Arc cRNA was transcribed from a full-length clone of the rat Arc gene subcloned into the pBluescript plasmid (Lyford et al., 1995). For nonradioactive in situ hybridization (NRISH), antisense cRNA probes for Arc were transcribed from linearized plasmid using T7 polymerase and synthesized using the Ambion (Austin, TX) Maxiscript in vitro transcription kit in the presence of digoxygenin-11-uridine-5 $\tilde{\mathrm{A}}$ triphosphate (Boehringer Mannheim, Indianapolis, IN). NRISH was performed as described previously (Paradies and Steward, 1997). For radioactive in situ hybridization, radiolabeled cRNA probes were prepared by in vitro transcription in the presence of $\left[{ }^{35} \mathrm{~S}\right]$-labeled uridine $5^{\prime}$ - $[\alpha$-thio $]$ triphosphate (NEN, Boston, MA), using the Stratagene (La Jolla, CA) RNA transcription kit. Sheet film autoradiograms were prepared by exposing the sections to $\beta$ max hyperfilm (Amersham Biosciences, Arlington Heights, IL) for $3 \mathrm{~d}$. The slides were then dipped in NTB2 emulsion (Eastman Kodak, Rochester, NY) and ex- posed for $7-8 \mathrm{~d}$ at $4^{\circ} \mathrm{C}$. Slides were developed using D19 (Eastman Kodak) and counterstained using cresyl violet.

Quantification. Granule cells that were heavily labeled for Arc were counted using a $20 \times$ objective from sections stained using the nonradioactive in situ hybridization procedure. The area of the dentate granule cell layer (GCL) in each section was determined using MCID M4 software system (Imaging Research, St. Catharines, Ontario, Canada), and cell counts were expressed the number of Arcpositive cells per square millimeter of tissue. Separate counts were taken for the dorsal and ventral blades of the GCL. Five to seven sections were analyzed for each animal.

The intensity of Arc mRNA labeling in CA1 and CA3 was measured from autoradiograms by measuring optical density (OD) using the MCID M4 software system. As an internal standard in each section, OD measurements were taken over the thalamus. Although strong sensory stimulation can induce IEG expression in specific nuclei, the behavioral paradigms here produced no detectable labeling of thalamic neurons, and so OD measurements in this region reflect background labeling. Three OD measurements were made in each region in three to five sections per animal. The OD values for each site were then averaged for each animal. A ratio value of region examined (CA1 or CA3) to thalamus (background control) was obtained for each animal.

Statistical analysis. The data from the cell counts from the dorsal and ventral blades of the dentate gyrus and ipsilateral and contralateral hippocampi were analyzed using betweengroups ANOVA. The two blades of the dentate gyrus were analyzed separately because qualitative evaluations suggested different responses (see Results). A Bonferroni correction was applied to control for type II error attributable to multiple comparisons. $p$ values reported for individual comparisons are after adjustment with the Bonferroni correction.

\section{Results}

\section{IEG induction after different types of} behavioral experience in intact animals In keeping with previous reports (Guzowski et al., 1999), the pattern of Arc expression seen depended on the animals' experience during the period immediately before death. Animals that remained isolated in their home cage exhibited low levels of Arc expression, whereas animals that were allowed to explore a novel complex environment or that had one or four training sessions in the MWM before death exhibited a dramatic upregulation of Arc expression throughout the forebrain. In the descriptions below, we will focus on the dentate gyrus and hippocampus, which receives its major extrinsic cortical input from the EC and loses this cortical input as a consequence of the lesions.

Figure 1 illustrates the patterns of Arc expression that were seen in isolated animals (Fig. 1A) and after the three types of behavioral experience (Fig. $1 B-D$ ). In the isolated animals, there were only light levels of labeling for Arc mRNA throughout most of the brain, with a small number of neurons standing out as having somewhat stronger levels of labeling. In the hippocampus, 
labeling for Arc mRNA was light throughout the CA1 and CA3 regions. The dentate gyrus exhibited a different pattern of labeling, in which most neurons showed no detectable labeling, whereas a few scattered cells exhibited high levels of labeling (Fig. 1) (see Fig. $4 A-D$, which provides higher-magnification views of the pattern of labeling in isolated animals). Levels of labeling were also low throughout the cerebral cortex (Fig. 2A).

In animals that either explored a novel complex environment for $1 \mathrm{hr}$ before death or experienced either 1 or $4 \mathrm{~d}$ of training in the MWM, Arc expression was strongly induced throughout the forebrain in a region- and cell type-specific manner. Their overall pattern of induction seen after the three types of experience was comparable, and so the description below applies to all three.

In the hippocampus, there was strong induction of Arc expression in CA1 and CA3. In the CA1 region, it appeared that most pyramidal cells exhibited increased labeling (Fig. $1 B-D$ ) (see Fig. 4D). There were also increases in the levels of labeling throughout the dendritic layers, consistent with the transport of newly synthesized Arc mRNA into dendrites (Fig. $1 B$, short arrows). In CA3, labeling was more sporadic; $\sim 30-50 \%$ of the pyramidal cells exhibited moderate to high levels of labeling, whereas the remainder exhibited low levels of labeling similar to what was seen in isolated animals (Fig. $1 B-D$ ) (see Fig. $4 E$ ). Dendritic labeling was less apparent in CA3 than CA1; indeed, there was a clear boundary of dendritic labeling at the CA1/CA2 junction (Fig. 1, long arrow).

In the dentate gyrus, there were a larger number of heavily labeled granule cells than in isolated animals, especially in the dorsal blade (Fig. $1 B-D$ ) (see Fig. $4 F$ ). Indeed, Arc expression seemed to be either "on" or "off" in individual granule cells. When granule cells exhibited any labeling at all, they were heavily labeled, and it was often possible to see labeled dendrites extending from the labeled granule cell bodies (see Fig. $4 F$ ). Except for the scattered granule cells that exhibited high levels of labeling, most granule cells exhibited no detectable labeling at all (see Fig. $4 F)$. In this sense, the overall levels of Arc expression in the dentate gyrus are reflected by what is essentially a digital readout: the number of granule cells exhibiting high levels of labeling.

Counts of the number of heavily labeled granule cells in the dorsal blade in the different groups (Fig. $1 E$ ) revealed a highly significant overall groups effect $\left(F_{(3,41)}=20.36 ; p<0.0001\right)$. Individual comparisons revealed that animals exposed to the complex environment or trained for one or four sessions in the MWM had a significantly larger number of labeled granule cells than animals kept in the home cage $(p<0.01, p<0.01$, and $p<$ 0.05 , respectively). There were no significant differences in the number of labeled granule cells between the "experience" groups, however. The counts from the ventral blade revealed that there were no significant differences between groups, confirming the impression from qualitative observations (Fig. $1 E$ ).

There was also a strong upregulation of Arc expression after exploration in many areas of the cerebral cortex (Fig. 2), as well as in other forebrain regions. In isolated animals, there were a few lightly labeled cells in layers I-VI (Fig. 2A), whereas in animals that were exposed to the complex environment, a large number of cells were labeled in all cortical layers, and there was a marked increase in the intensity of labeling of individual cells (Fig. $2 B$ ).

Because the extent and pattern of Arc induction was generally comparable after the three types of experience, we chose to evaluate the consequences of brain injury using the task involving exposure to the novel complex environment. The reason is that it is simpler to allow animals to explore than it is to train animals in
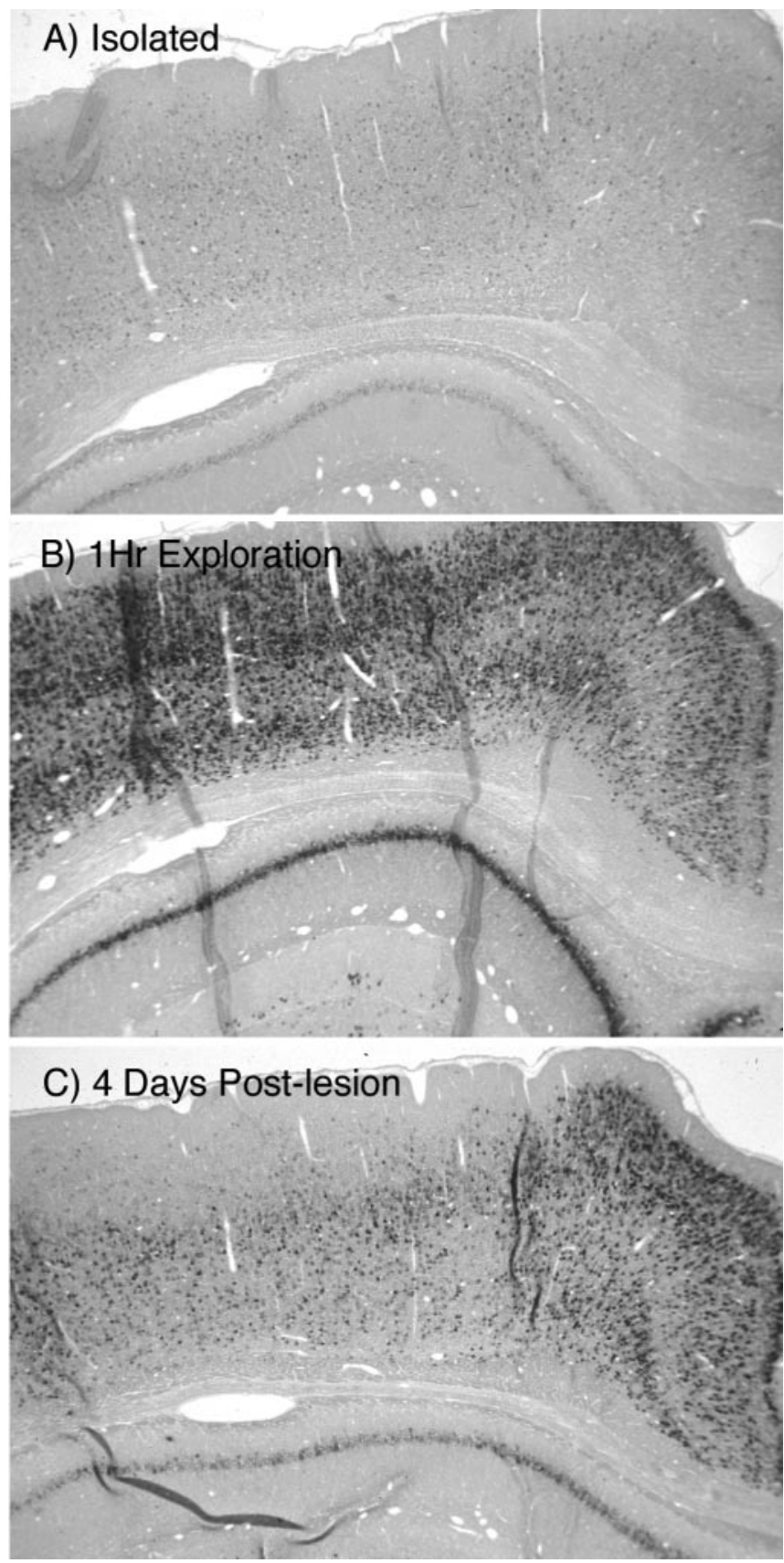

Figure 2. Patterns of expression of Arc mRNA in the cortex. $A$, Control animals left undisturbed in their home cage (isolated controls). $B$, After $1 \mathrm{hr}$ of exploration of a novel complex environment. C, Animal killed $4 \mathrm{~d}$ after a unilateral $\mathrm{EC}$ lesion after $1 \mathrm{hr}$ of exploration of a novel complex environment.

the Morris water maze, increasing the utility of the task as a standard assessment tool.

\section{Changes in Arc expression after lesions}

Lesion verification

The extent of the lesion in the EC was evaluated in horizontal sections stained with cresyl violet, and the extent of the denervation in the molecular layer of the dentate gyrus was assessed using AChE histochemistry. Lesions of the EC result in a band of increased AChE staining corresponding to the portion of the molecular layer that is denervated (Cotman and Nadler, 1978). Lesions involving medial and lateral portions of the EC result in 

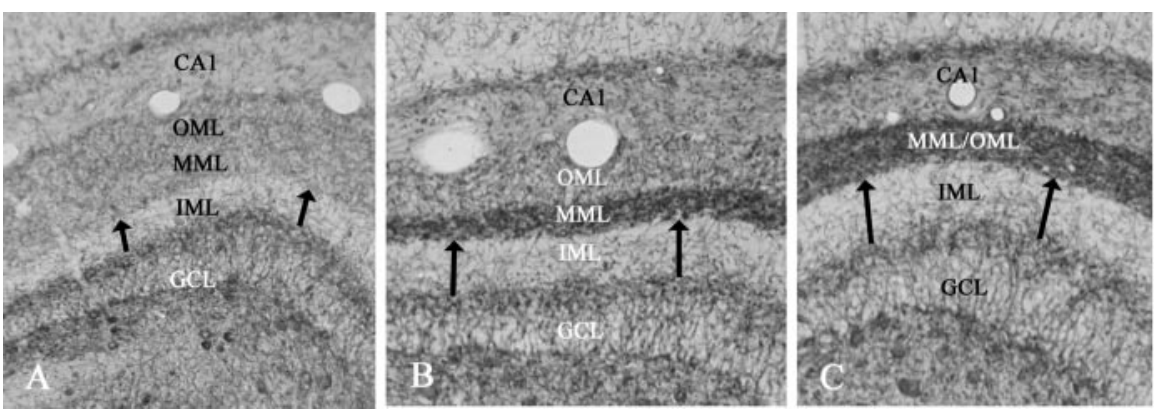

Figure 3. AChE staining reveals the extent of denervation in the dentate gyrus. $A$, Pattern of $A C h E$ staining in control animals. $B$ and $C$ illustrate increases in AChE staining in the denervated neuropil of the dentate gyrus in animals killed $30 \mathrm{~d}$ after lesion. $B$ Selective increases in AChE staining in the middle molecular layer of the dentate gyrus (indicative of a lesion that involved only the medial portion of the entorhinal cortex). C, Increases in AChE staining throughout the middle and outer molecular layer (indicative of a lesion that involved both medial and lateral portions of the EC). Note the increases in the width of the AChE-free zone in the inner molecular layer, which is related to the expansion of the terminal field of the dentate commissural system. GCL, Granule cell layer; $I M L$, inner molecular layer; $M M L$, middle molecular layer; $O M L$, outer molecular layer; $C A 1$, distal dendritic region of $C A 1$. Scale bar, $250 \mu \mathrm{m}$
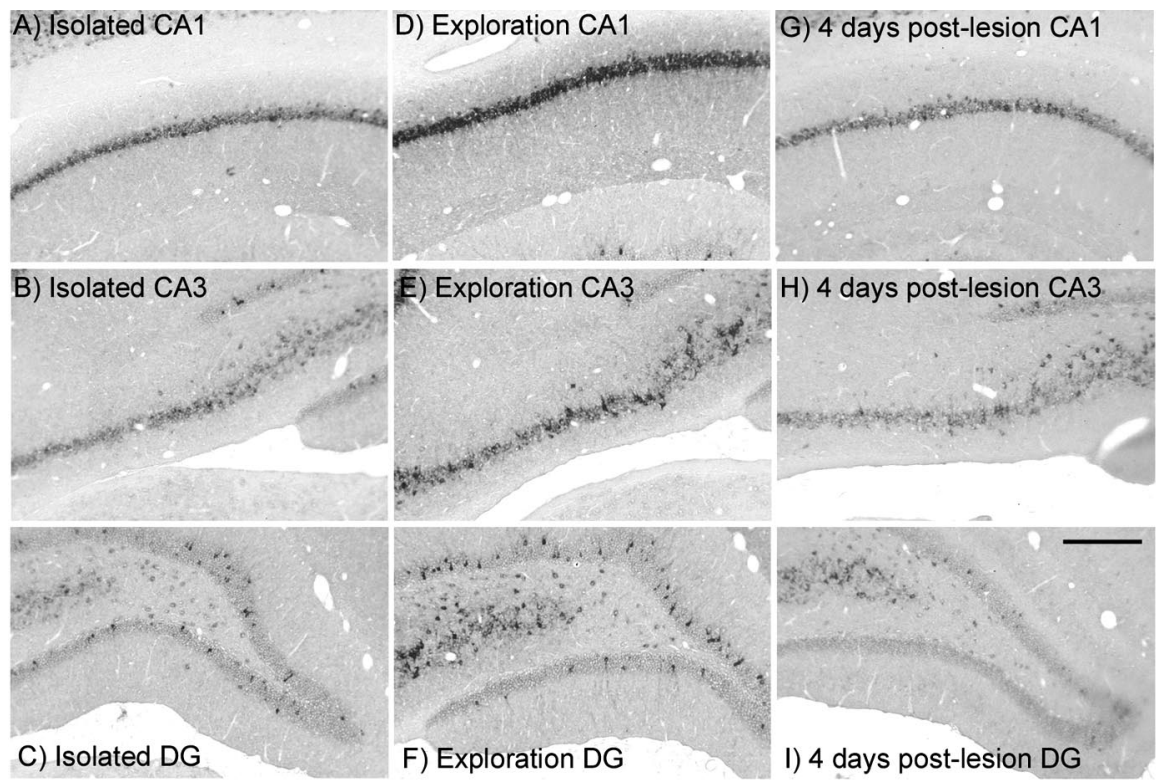

C) Isolated DG

Figure 4. Induction of Arc expression in different subdivisions of the hippocampus after a 1-hr-long period of exploration of a novel complex environment in unoperated animals and at various times after a unilateral EC lesion. The photomicrographs illustrate Arc mRNA distribution as revealed by nonisotopic in situ hybridization. $A-C$, Control animals left undisturbed in their home cage (isolated controls). D- $F$, Unoperated animal after $1 \mathrm{hr}$ of exploration of a novel complex environment. $G-I$, Animal killed $4 \mathrm{~d}$ after a unilateral $\mathrm{EC}$ lesion after $1 \mathrm{hr}$ of exploration of a novel complex environment. CA1 and CA3 indicate the respective subdivisions of the hippocampus proper. DG, Dentate gyrus. Scale bar, $250 \mu \mathrm{m}$.

increases in AChE staining throughout the middle and outer molecular layers of the dentate gyrus. Selective lesions of the medial EC result in a discrete band of increased AChE staining in the middle molecular layer. The lesions in the present study were of both types (Fig. 3), and, at each survival interval, there were animals that had complete or selective medial lesions (Table 1). Animals with either complete or selective medial lesions exhibited qualitatively similar changes in Arc expression, and so the two sets were combined for quantitative analysis.

Arc expression is depressed at early postlesion intervals

At early time points after unilateral EC lesions, basal and experience-induced Arc expression was dramatically depressed. In what follows, we illustrate this effect in the animals that were exposed to the novel environment, but the quantitative analysis (see below) compared both basal expression and expression induced by experience across groups.

Figure 4 illustrates the dramatic depression of experience-induced Arc expression at early postlesion intervals. Figure $4 A-C$ illustrates the pattern of Arc expression in an animal that was isolated in its home cage, Figure $4 D-F$ illustrates the pattern of expression in an unoperated animal that had explored the complex environment for $1 \mathrm{hr}$, and Figures $4 G-I$ and 5 illustrate the pattern of expression in animals that were exposed to the novel environment at various postlesion intervals. At $4 \mathrm{~d}$ after lesion (Fig. $4 G-I$ ), Arc expression was depressed in all subregions of the hippocampus proper ipsilateral to the lesion. Indeed, the overall extent of labeling appeared to be reduced even compared with unoperated animals that had been isolated in their home cages. There was minimal labeling in either CA1 or $\mathrm{CA} 3$, and there were few if any granule cells exhibiting labeling (Fig. 4I).

To quantitatively assess the changes in Arc expression, we counted the number of heavily labeled granule cells in the dorsal and ventral blades of the dentate gyrus. The two blades were assessed separately because the experience-induced increases in the number of labeled granule cells occurs primarily in the dorsal blade (see above). The unoperated control groups prepared to assess the consequences of lesions provided an opportunity to replicate the experiment assessing IEG expression in control animals and animals that were briefly exposed to a novel complex environment. As illustrated in Figure $6 \mathrm{~A}$, we again saw a striking increase in the number of Arc-positive cells in the dorsal blade of the GCL after exposure to the novel complex environment $\left(F_{(14,14)}=8.51\right.$; $p=0.0006)$. In contrast, there was no significant difference in the number of Arcpositive cells between isolated and exploration conditions in the ventral blade $\left(F_{(14,14)}=1.21 ; p=0.721\right)$ (Fig. $\left.6 C\right)$.

Counts of the numbers of heavily labeled granule cells in animals that had been exposed to the novel complex environment at 4 or $8 \mathrm{~d}$ after lesion revealed a complete blunting of the experience-induced increases in the number of labeled granule cells (Fig. 6A). Also, fewer labeled granule cells were seen in the isolated animals at 4 and $8 \mathrm{~d}$ after lesion (Fig. $6 \mathrm{~A}$, dorsal blade, $C$, ventral blade). Two separate ANOVAs were performed to evaluate the numbers of labeled granule cells in dorsal and ventral blades; both ANOVAs revealed significant overall main effects (dorsal blade, $F_{(11,75)}=23.33, p<0.0001$ ); ventral blade, $\left(F_{(11,76)}=17.93, p<0.000 .1\right)$. Post hoc comparisons revealed that there were significantly fewer labeled granule cells in both dorsal and ventral blades in the animals that had been exposed to the novel complex environment at all postlesion time points except $30 \mathrm{~d}$ ( $p<0.01$ for all time points). 
There were also significantly fewer labeled granule cells in both blades at all postlesion intervals in the operated animals that remained isolated in their home cage (Fig. 6, open symbols). This was true in both dorsal and ventral blades of the dentate gyrus (Fig. 6A, $C$, for dorsal and ventral blades, respectively).

Two questions arise when considering the decreased IEG expression after lesions. The first is whether the operated animals in fact explored the novel environment when presented the opportunity to do so. Although we did not systematically assess the animals' behavior in the open field during the period of exploration, the animals did seem to exhibit the same general behavior as unoperated animals. They certainly did not remain motionless. Importantly, previous studies demonstrated that animals with unilateral EC lesions exhibit comparable levels of exploratory activity compared with control animals when exposed to a novel environment (Steward et al., 1977). Thus, it seems that it is the responsiveness of the brain (in terms of IEG expression) that is different in operated animals and not the animals' activity in the novel environment.

The second question is whether decreases in Arc expression might be a long-term result of the anesthetic. To address this question, we assessed experience-induced Arc expression in animals that were anesthetized and received sham lesions. As illustrated in Figure $8 \mathrm{~A}$, animals that had received "sham" lesions exhibited the same patterns of experience-induced Arc expression as control animals.

\section{Arc expression recovers over time}

Comparisons of Arc expression at various postlesion intervals revealed a time-dependent recovery in the levels of experienceinduced Arc expression after unilateral EC lesions that was first apparent at $12 \mathrm{~d}$ after lesion and increased progressively thereafter (Fig. 5). By $16 \mathrm{~d}$ after lesion, the levels of activity-induced expression in the hippocampus appeared qualitatively similar to that seen in unoperated animals (Fig. $5 D-F$ ), and levels of expression were maintained at $30 \mathrm{~d}$ after lesion (Fig. $5 E-G$ ). The quantitative analysis of numbers of labeled granule cells confirmed the qualitative impressions (Fig. 6). By $30 \mathrm{~d}$ after lesion, animals that had been exposed to the novel complex exhibited as many labeled granule cells in the dorsal blade as the control animals (Fig. 6A). The extent of recovery was less in the ventral blade but was still detectable (Fig. 6C).

Interestingly, although there was a recovery of experienceinduced expression, the quantitative analysis of numbers of Arcpositive granule cells revealed that this aspect of Arc expression remained depressed in isolated animals, even at $30 \mathrm{~d}$ after lesion (Fig. 6, open squares).

Arc expression is also depressed at early postlesion intervals in the dentate gyrus contralateral to the lesion

As noted above, unoperated animals exhibit a consistent pattern of labeling in the dentate gyrus in which scattered neurons exhibit high levels of labeling. This pattern of labeling was also disrupted in the hippocampus contralateral to the lesion and also in the contralateral dentate gyrus, which receives only a very sparse projection that is restricted to the anterior pole of the dentate gyrus (Goldowitz et al., 1975). Figure 7B illustrates an example of depressed expression at $8 \mathrm{~d}$ after lesion in an animal that had been exposed to the novel complex environment. The quantitative assessment of numbers of labeled granule cells in the dentate gyrus contralateral to the lesion is shown in Figure 6, $B$ and $D$. Levels of expression were substantially depressed in the contralateral dentate gyrus at 4 and $8 \mathrm{~d}$ after lesion and then recovered with approximately the same time course as on the ipsilateral side. ANOVAs comparing the number of labeled granule cells revealed significant main effects (dorsal blade, $F_{(11,76)}=14.51, p<0.0001$; ventral blade, $\left.F_{(11,76)}=8.41, p<0.0001\right)$.

In the dorsal blade of the dentate gyrus contralateral to the lesion (Fig. 6C), there were significantly fewer labeled granule cells in the animals that had been exposed to the novel complex environment at all postlesion time points except $30 \mathrm{~d}(p<0.05$ at day 12 and $p<0.01$ for 4,8 , and $16 \mathrm{~d}$ ). In the case of the animals that remained isolated in their home cage, post hoc comparisons revealed a significant difference between control and the $8 \mathrm{~d}$ time point and no significant differences at other postlesion intervals.

In the ventral blade (Fig. 6D), the quantitative analysis revealed that there were significantly fewer labeled granule cells at 4 and $8 \mathrm{~d}$ after lesion in animals that had experienced the complex environment $(p<0.01)$. There were no significant differences between the unoperated controls and the other postlesion time points for either the animals that were exposed to the novel environment or the animals that remained isolated in their home cage.

\section{Quantitative assessment of Arc expression in the hippocampus proper}

The quantitative assessment of Arc expression in the dentate gyrus takes advantage of what is essentially a digital readout; Arc expression appears to be either "on" or "off" in individual granule cells, and so one can count the number of labeled granule cells. In the hippocampus, especially CA1, changes in Arc expres- 

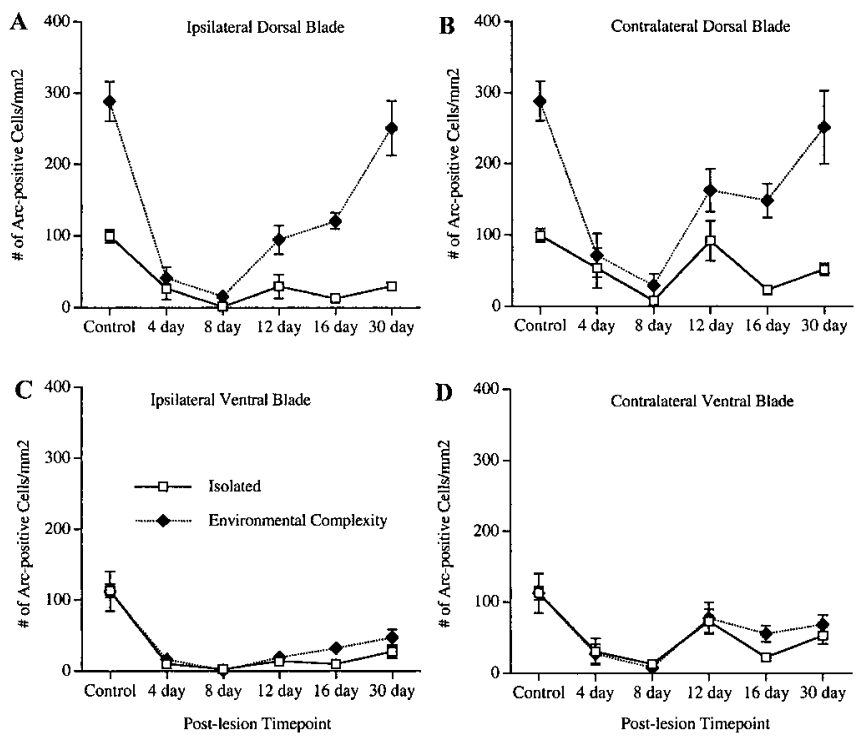

Figure 6. Quantitative assessment of the numbers of Arc-positive granule cells in the dentate gyrus after exposure to the novel environment at various postlesion intervals. $A$, Counts of Arc-positive granule cells in the dorsal blade of the dentate gyrus ipsilateral to the lesion. $B$, Counts of Arc-positive granule cells in the dorsal blade of the dentate gyrus contralateral to the lesion. C, Counts of Arc-positive granule cells in the ventral blade of the dentate gyrus ipsilateral to the lesion. D, Counts of Arc-positive granule cells in the ventral blade of the dentate gyrus contralateral to the lesion.

sion attributable to experience appear to involve most pyramidal neurons. Accordingly, to quantify changes in Arc expression in the hippocampus, sections were prepared for radioactive in situ hybridization and film autoradiography, and levels of expression were quantified by measuring relative OD in the film autoradiographs. Relative OD in this case refers to the ratio of OD in the respective region versus the thalamus, which serves as an internal standard in which Arc is expressed at minimal levels.

In unoperated animals, exposure to the novel environment led to an approximate twofold increase in relative OD in CA1 (Fig. 7A). At the same time, there was only a slight increase in relative OD in CA3 (Fig. 7C). The experience-induced increases in relative OD in CA1 ipsilateral to the lesion were not seen at 4 and $8 \mathrm{~d}$ postlesion but reappeared at longer postlesion intervals (Fig. 7A). In the CA1 region contralateral to the lesion, experience-induced increases were seen at all postlesion time points except $8 \mathrm{~d}$ (Fig. 7B). Given that experience-induced increases in Arc expression are very slight in CA3 in unoperated animals, it is probably not meaningful to place much emphasis on changes after lesions. Nevertheless, the slight increases in relative $\mathrm{OD}$ that were seen in CA3 in unoperated animals after exposure to the novel environment were not seen at 8 and $12 \mathrm{~d}$ after lesion and reappeared at longer postlesion intervals.

\section{Arc expression is also depressed after bilateral lesions of the entorhinal cortex}

It was of interest to assess Arc expression after bilateral EC lesions because animals with bilateral lesions show increased levels of exploratory activity when exposed to a novel environment compared with unoperated control animals (Steward et al., 1977). Thus, if animals with bilateral EC lesions exhibit decreases in Arc expression, it is not because the animals are less active. As illustrated in Figure $8 B$, Arc expression was strikingly depressed in the hippocampus $4 \mathrm{~d}$ after a bilateral $\mathrm{EC}$ lesion. This is at a time that animals exhibit peak levels of exploratory activity in a novel environment (Steward et al., 1977).
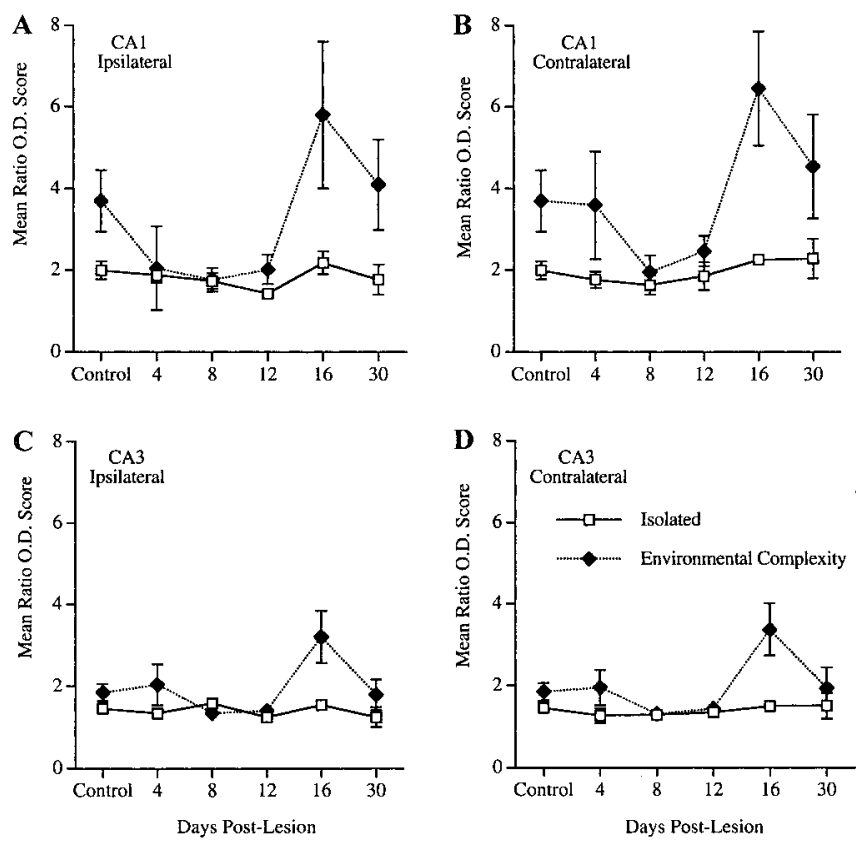

Figure 7. Arc expression in the $C A 1$ and $C A 3$ regions after unilateral lesions of the $E C$ as measured by radioactive in situ hybridization and film autoradiography. The graphs illustrate the mean $0 \mathrm{D}$ in $\mathrm{CA} 1$ and $\mathrm{CA} 3$ (normalized by an internal standard as described in Materials and Methods) at various postlesion intervals.

\section{Arc expression is also depressed in widespread cortical areas}

There were also dramatic decreases in labeling in the cerebral cortex at early time points after EC lesions (Fig. 2C). These decreases in labeling occurred differentially in various cortical regions. For example, experience-induced Arc expression in the cingulate cortex remained high in the operated animals, whereas labeling in the laterally adjacent regions of the neocortex was depressed. We did not evaluate these interesting patterns of Arc expression in the cortex in detail because our focus was the hippocampus, in which the lesion-induced synaptic loss and replacement have been well characterized. Nevertheless, the decreases in experience-induced Arc expression in operated animals are consistent with widespread depression of experience-induced neuronal activity as a result of the lesion.

\section{Arc expression is also depressed after unilateral}

fimbria-fornix lesions

The fact that Arc expression is depressed after unilateral EC lesions and then recovers implies that the entorhinal cortex plays a key role in relaying the activity that is necessary for experienceinduced Arc expression. Thus, it was of interest to assess whether other input pathways, which convey different types of information, also played a role in Arc expression. Thus, we evaluated experience-induced Arc expression in animals that had received unilateral lesions of the fimbria-fornix.

Figure 9 illustrates the pattern of Arc expression in the posterior hippocampus contralateral (Fig. 9A) and ipsilateral to the fimbria-fornix lesion (Fig. 9B). For these experiments, brains were sectioned in the horizontal plane to allow lesion verification. On the side ipsilateral to the lesion, levels of Arc mRNA were very low throughout most of CA1 and CA3, and there were very few labeled granule cells. Despite the virtual absence of Arc expression in the hippocampus proper, the levels of Arc expression were quite high in the adjacent cerebral cortex and even in the posterior subiculum. Interestingly, levels of Arc expression appeared 

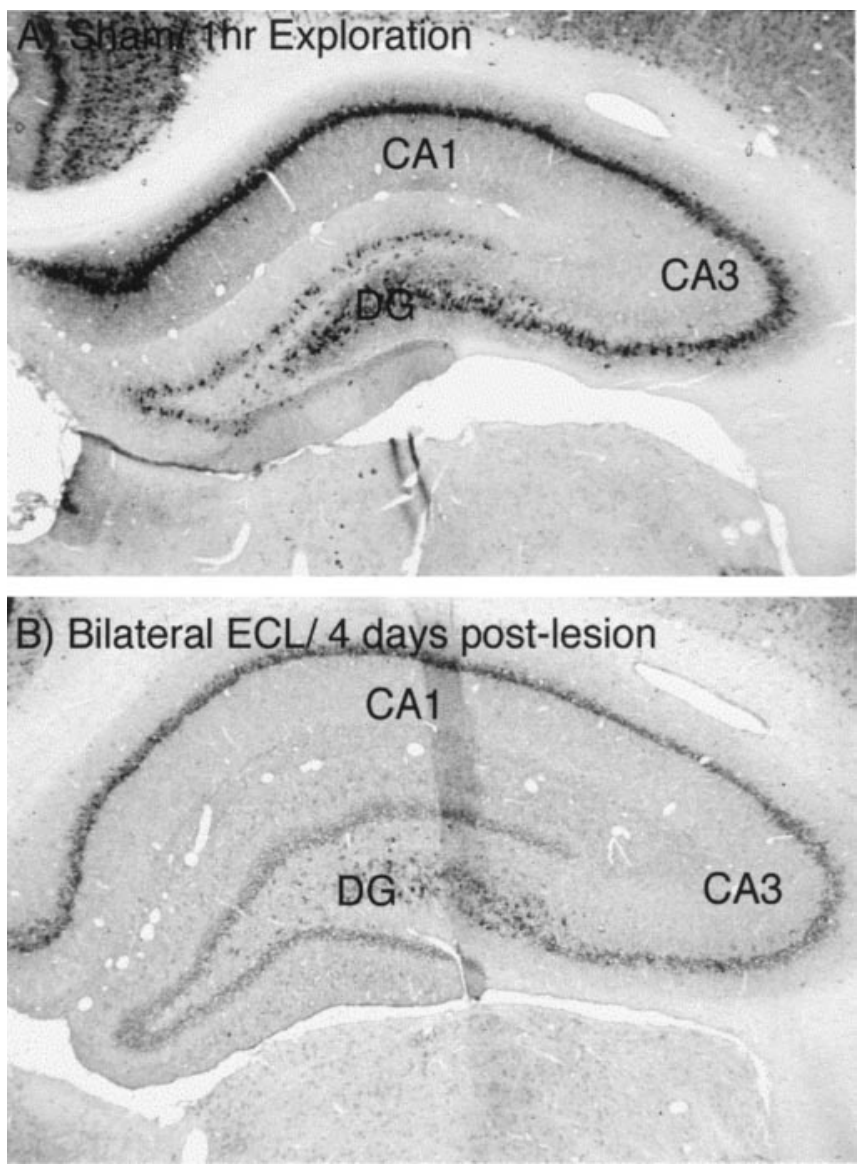

Figure 8. Arc expression after sham lesions and after bilateral EC lesions. A, Pattern of Arc expression $4 \mathrm{~d}$ after a sham lesion in an animal that was allowed to explore a novel environment for $1 \mathrm{hr}$ before death. B, Pattern of Arc expression $4 \mathrm{~d}$ after a bilateral EC lesion (ECL) in an animal that was allowed to explore a novel environment for $1 \mathrm{hr}$ before death. DG, Dentate gyrus.

normal in the hippocampus and dentate gyrus contralateral to the fimbria-fornix lesion (Fig. 9A).

\section{Discussion}

The present study evaluated whether experience-induced IEG expression could be used to define the ensemble of neurons that were activated by experience in brains in which connections had been modified as a consequence of injury. Our results revealed striking time-dependent alterations in experience-induced IEG expression after unilateral lesions of the entorhinal cortex. At early postlesion intervals, experience-induced IEG expression was profoundly depressed in areas that would have been denervated by the lesions (the hippocampus ipsilateral to the lesion), and also in neurons that receive a more minor input from the entorhinal cortex, and are downstream from the dentate granule cells (pyramidal neurons in CA3 and CA1). Over time, there was a recovery in experience-induced IEG expression that occurred over the same period of time in which neurons are being reinnervated. There were also global decreases in experience-induced IEG induction throughout the cerebral cortex and in the contralateral dentate gyrus, which retained almost all of its normal innervation. These results suggest that widespread alterations in IEG expression reflect changes in activity over polysynaptic circuits that would normally be engaged during the experience.

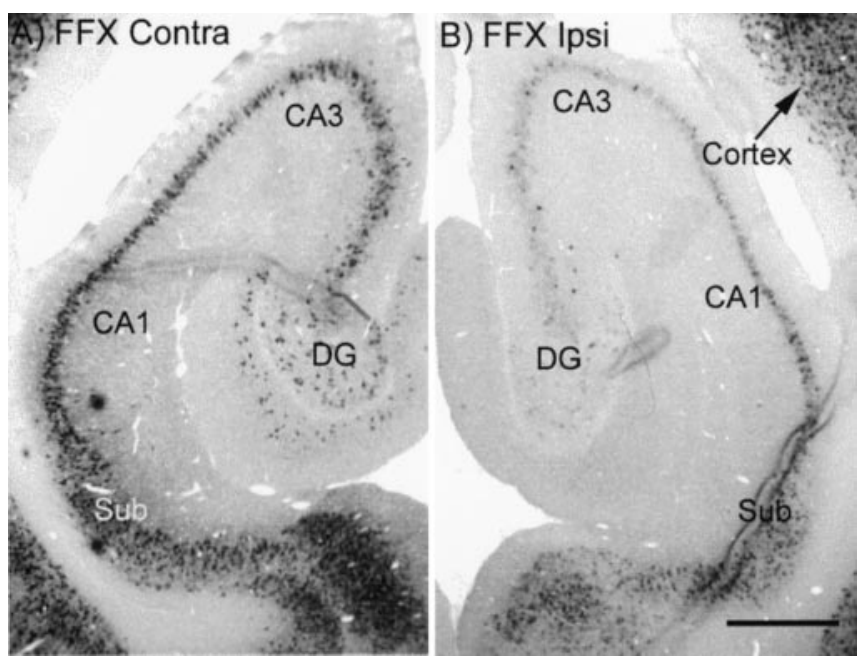

Figure 9. Arc expression is depressed in the hippocampus after unilateral lesions of the fimbria-fornix. A, Pattern of Arc expression as revealed by nonisotopic in situ hybridization in the posterior hippocampus contralateral to the fimbria-fornix lesion (FFX Contra). B, Pattern of expression ipsilateral to the lesion (FFXIpsi). Brains were sectioned in the horizontal plane so as to confirm the completeness of the lesion. Note low levels of Arc expression in the hippocampus and dentate gyrus $(D G)$ and the high levels of expression in the adjacent cerebral cortex (Cortex) and the posterior subiculum (Sub).

\section{What does the pattern of IEG induction signify in terms of activity of individual neurons?}

Exploration in a novel environment strongly induces Arc expression in a variety of forebrain structures. During the period of exploration, animals actively explore the environment, interacting with the various toys that are present, whereas animals that are isolated in their home cage experience no novel stimuli. Obviously, there must be substantial differences in ensemble neuronal activity in the different settings. Given that Arc induction depends critically on NMDA receptor activation (Lyford et al., 1995), high levels of Arc expression may mark the population of neurons whose NMDA receptors have been sufficiently activated during the period before death. An interesting corollary of this conclusion derives from the fact that NMDA receptor activation is the key step for triggering synaptic modifications such as longterm potentiation. Thus, Arc induction may reflect those neurons that have undergone synaptic activation that is sufficient to trigger synaptic modifications.

\section{Decreases in Arc expression after lesions indicate a widespread depression of circuit activity}

Both basal and experience-induced Arc expression were profoundly depressed at early postinjury intervals. These decreases in experience-induced expression were seen in the dentate gyrus ipsilateral to the lesion, in which granule cells lost $\sim 80 \%$ of their excitatory innervation, in CA1 and CA3 pyramidal neurons, which receive a minor projection from the $\mathrm{EC}$, in the dentate gyrus contralateral to the lesion, which receives only a very tiny projection from the EC, and in areas of the cerebral cortex ipsilateral to the lesion. How do these changes in labeling correlate with changes in ensemble neuronal activity? In the case of the dentate granule cells ipsilateral to the lesion, this question can be answered in some detail. Studies of changes in "spontaneous" neuronal activity after EC lesions (Reeves and Steward, 1988) reveal that "spontaneous" activity in anesthetized animals decreases by $\sim 80 \%$ at early postlesion intervals and then recovers with reinnervation. 
For the other cell populations, no such comparative physiological data are available. One might predict that the removal of EC input would cause a less dramatic change in neuronal activity in CA3, CA1, and the cerebral cortex because these areas receive a far smaller proportion of their total excitatory input from the entorhinal cortex. Indeed, in the case of CA1, there is evidence that input from the EC (via the temporo-ammonic pathway) produces a net inhibitory effect on CA1 pyramidal neurons because the pathway strongly activates inhibitory interneurons, producing feedforward inhibition (Empson and Heinemann, 1995; Lacaille and Schwartzkroin, 1988). Another line of reasoning predicts the opposite, based on the fact that the dentate gyrus is the first stage of the so-called trisynaptic circuit, which relays information from the EC through the dentate gyrus (via the perforant path), to CA3 (via the mossy fibers), and on to CA1 (via the Schaffer collateral system). The profound decrease in activity at the first stage of the circuit (the dentate gyrus) would reduce excitatory synaptic drive of downstream cell populations (in CA3 and CA1).

The dramatic decreases in IEG expression after EC lesions indicate that $\mathrm{EC}$ lesions have a more dramatic effect on neuronal activity than would be predicted by the direct loss of excitatory input to the respective cell populations. In this regard, it is interesting to again consider the hypothesis that Arc expression is more closely linked to NMDA receptor activation than to postsynaptic activity per se (that is, the rate of action potential generation). If this is so, modest decreases in synaptic drive could reduce the overall level of depolarization of the affected neuronal populations, shifting the membrane potential farther away from the threshold for NMDA receptor activation. In this way, a small change in excitatory synaptic drive could produce a large change in Arc expression.

\section{Relationship between recovery of Arc expression, circuit reorganization and reinnervation, and behavioral recovery} The initial depression of Arc expression and the time-dependent recovery are consistent with the interpretation that denervation disrupts functional activation in widespread circuitry, and reinnervation restores that activity. By $16 \mathrm{~d}$ after lesion, there is substantial reinnervation of dentate granule cells (Steward and Vinsant, 1983), a virtually complete restoration of normal levels of spontaneous neuronal activity (Reeves and Steward, 1988), substantial behavioral recovery in tasks that require the hippocampus (Loesche and Steward, 1977), and a near-normal pattern of experience-induced Arc activation. Of course, these are correlations, and definitive evidence for a causal relationship is lacking.

Although there was a recovery in experience-induced Arc expression after EC lesions, basal levels of expression remained depressed. This may indicate that the overall level of basal circuit activity remains depressed.

\section{Arc expression after brain lesions: a way to visualize diaschisis?}

The term diaschisis was coined by von Monakow (1969) to refer to a depression of function or loss of responsiveness in brain regions that are connected to regions that are damaged by lesions. von Monakow envisioned a phenomenon similar to the "spinal shock" that is seen after spinal cord injury, in which there is an overall depression of reflex activity and a loss of responsiveness. "In ... diaschisis, the ability to respond to stimuli ... becomes impaired, abolished or refractory" (von Monakow, 1969). von
Monakow proposed that loss of function after brain injury was attributable to both the actual damage and depression of function in regions that were connected to the damaged region and that the resolution of diaschisis was an important reason for recovery after injury.

The global decreases in basal and experienced-induced Arc expression that we describe here are exactly the sorts of changes that von Monakow envisioned. Widespread decreases in expression were seen in areas that are downstream from the damaged structure, and there was a recovery of Arc expression over approximately the same time period as the recovery of function that has been documented in previous studies (for review, see Steward, 1982).

It should be noted that there have been previous experimental tests of diaschisis after unilateral EC lesions (West et al., 1976). This experiment evaluated synaptic responses generated in denervated dentate granule cells by stimulation of surviving pathways (specifically, the pathway from the contralateral hippocampus). Interestingly, there was no evidence of diminished synaptic potency at early postlesion intervals as might be predicted by the hypothesis of diaschisis. The present results demonstrate that, although evoked responses generated by surviving synaptic inputs are not depressed, the loss of synaptic drive from the entorhinal cortex depresses whatever aspect of postsynaptic activity is reflected by increased Arc expression.

The profound changes in activity-dependent IEG expression document a novel tool to evaluate functional recovery after brain injury, providing a measure of functional activity in ensembles of neurons engaged in a behavioral experience. IEG expression may reflect a threshold level of activation of NMDA receptors sufficient to trigger Hebb-like synaptic modifications. In this regard, Arc induction may provide a convenient measure of the loss and restoration of a level of functional activity required for higher cognitive function, which could explain general decrements in cognitive function after brain injury. It will be of considerable interest to assess IEG modulation in other models of brain injury to further explore this hypothesis.

\section{References}

Cotman C, Nadler JV (1978) Reactive synaptogenesis in the hippocampus (Cotman CW, ed). New York: Raven.

Empson RM, Heinemann U (1995) Perforant path connections to area CA1 are predominantly inhibitory in the rat hippocampal-entorhinal cortex combined slice preparation. Hippocampus 5:104-107.

Goldowitz D, White WF, Steward O, Lynch G, Cotman CW (1975) Anatomical evidence for a projection from the entorhinal cortex to the contralateral dentate gyrus of the rat. Exp Neurol 47:433-441.

Guzowski JF, McNaughton BL, Barnes CA, Worley PF (1999) Environmentspecific induction of the immediate early gene Arc in hippocampal neuronal ensembles. Nat Neurosci 2:1120-1124.

Kelly MP, Deadwyler S (2002) Acquisition of a novel behavior induces higher levels of Arc mRNA than does overtrained performance. Neuroscience 110:617-626.

Lacaille J-C, Schwartzkroin PA (1988) Stratum lacunosum-moleculare interneurons of hippocampal CA1 region. II. Intrasomatic and intradendritic recordings of local circuit interactions. J Neurosci 8:1411-1424.

Loesche J, Steward O (1977) Behavioral correlates of denervation and reinnervation of the hippocampal formation of the rat: recovery of alternation performance following unilateral entorhinal cortex lesions. Brain Res Bull 2:31-39.

Lyford GL, Yamagata K, Kaufmann WE, Barnes CA, Sanders LK, Copeland NG, Gilbert DJ, Jenkins NA, Lanahan AA, Worley PF (1995) Arc, a growth factor and activity-regulated gene, encodes a novel cytoskeletonassociated protein that is enriched in neuronal dendrites. Neuron 14:433-445. 
Matthews DA, Cotman C, Lynch G (1976a) An electron microscopic study of lesion-induced synaptogenesis in the dentate gyrus of the adult rat. I. Magnitude and time course of degeneration. Brain Res 115:1-21.

Matthews DA, Cotman C, Lynch G (1976b) An electron microscopic study of lesion-induced synaptogenesis in the dentate gyrus of the adult rat. II. Reappearance of morphologically normal synaptic contacts. Brain Res 115:23-41.

Paradies MA, Steward O (1997) Multiple subcellular mRNA distribution patterns in neurons: a nonisotopic in situ hybridization analysis. J Neurobiol 33:473-493.

Phillips LL, Lyeth BG, Hamm RJ, Povlishock JT (1994) Combined fluid percussion brain injury and entorhinal cortical lesion: a model for assessing the interaction between neuroexcitation and deafferentation. J Neurotrauma 11:641-656.

Pinaud R, Penner MR, Robertson HA, Currie RW (2001) Upregulation of the immediate early gene Arc in the brains of rats exposed to environmental enrichment: implications for molecular plasticity. Brain Res Mol Brain Res 91:50-56.
Reeves T, Steward O (1988) Changes in the firing properties of neurons in the dentate gyrus with denervation and reinnervation: significance for behavioral recovery. Exp Neurol 102:37-49.

Steward O (1982) Assessing the functional significance of lesion-induced neuronal plasticity. Int Rev Neurobiol 23:197-254.

Steward O (1989) Reorganization of neuronal connections following CNS trauma: principles and experimental paradigms. J Neurotrauma 6:99-152.

Steward O, Vinsant SL (1983) The process of reinnervation in the denate of the adult rat: a quantitative electron microscopic analysis of terminal proliferation and reactive synaptogenesis. J Comp Neurol 214:370-386.

Steward O, Loesche J, Horton WC (1976) Behavioral correlates of denervation and reinnervation of the hippocampal formation of the rat: open field activity and cue utilization following bilateral entorhinal cortex lesions. Brain Res Bull 2:41-48.

von Monakow C (1969) Diaschisis. In: Mood, states, and mind (Pribram K, ed), pp 68-94. Baltimore: Penguin.

West JR, Deadwyler SA, Cotman CW, Lynch GS (1976) An experimenta test of diaschisis. Behav Biol 18:419-425. 